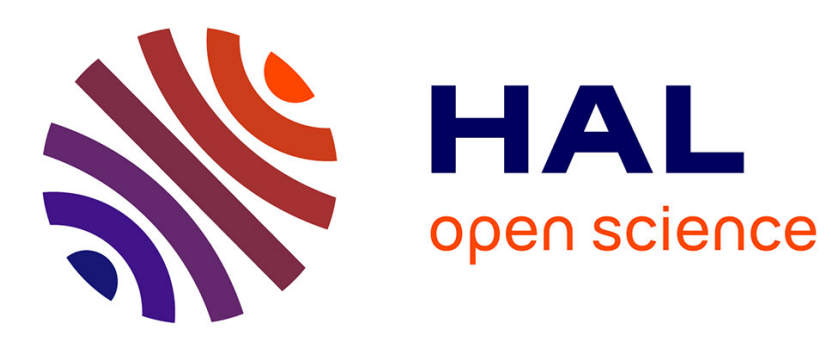

\title{
Some examples of path following in microrobotics
}

Bassem Dahroug, Jean Seon, Brahim Tamadazte, Nicolas Andreff, Ali

Oulmas, Tiantian Xu, Stéphane Regnier

\section{To cite this version:}

Bassem Dahroug, Jean Seon, Brahim Tamadazte, Nicolas Andreff, Ali Oulmas, et al.. Some examples of path following in microrobotics. International Conference on Manipulation, Automation and Robotics at Small Scales, Jul 2018, Nagoya, Japan. hal-02392507

\section{HAL Id: hal-02392507 \\ https://hal.science/hal-02392507}

Submitted on 4 Dec 2019

HAL is a multi-disciplinary open access archive for the deposit and dissemination of scientific research documents, whether they are published or not. The documents may come from teaching and research institutions in France or abroad, or from public or private research centers.
L'archive ouverte pluridisciplinaire HAL, est destinée au dépôt et à la diffusion de documents scientifiques de niveau recherche, publiés ou non, émanant des établissements d'enseignement et de recherche français ou étrangers, des laboratoires publics ou privés. 


\section{Some examples of path following in microrobotics}

\author{
Bassem Dahroug, Jean-Antoine Séon \\ Brahim Tamadazte, Nicolas Andreff \\ Institut FEMTO-ST \\ Univ. Bourgogne Franche-Comté / CNRS \\ Besançon, France \\ firstname.lastname@femto-st.fr
}

\author{
Ali Oulmas, Tiantian Xu \\ Stéphane Régnier \\ Institut des Systèmes Intelligents et Robotique (ISIR) \\ Sorbonne Universités, UPMC University Paris 06 \\ Paris, France \\ firstname.lastname@isir.upmc.fr
}

\begin{abstract}
After reviewing the various motion generation strategies (waypoint sequence, trajectory tracking and path following), this paper suggests that path following is probably the best strategy at small scales. This is illustrated by three examples coming from biomedical applications: endoscopic laser steering, magnetic manipulation of microswimmers and navigation of a surgical tool under anatomical constraints.
\end{abstract}

Index Terms-microrobot control, biomedical robotics, path following, visual servoing, laser steering, magnetic manipulation

\section{INTRODUCTION}

Small scale creates specific challenges to robotics related to a different ratio between volumic forces (namely, gravity and dynamics) and surface forces (namely, friction) than at conventional scale [1]. One of them is related to motion control. Indeed, at small scale, inertia is often neglectable, which has two effects. The first one is that small objects can reach a very high velocity almost immediately, the second one is that friction (stiction at the solid/air interface or fluidic drag) becomes a predominant disturbance. Therefore, the conventional motion control strategies might have to be reconsidered.

This issue was encountered when tuning the controller for the MagPier, on the occasion of the NIST mobility challenge at ICRA 2011 [2]. Indeed, the mobility challenge consisted in a slalom through 4 gates, and the MagPier was a magnetic particle driven by a tunable magnetic field. Therefore, a "trajectory" (see below for a proper definition) passing through the gates was defined and conventional robot control was applied, but it would not work.

What happened ? In fact, the trajectory was defined as a sequence of waypoints and designed the controller as a point to point step response, with a simple PID control providing the force towards the next waypoint, this force being then converted into currents into the magnetic coils by appropriate transduction. Thereby, the MagPier would stay stuck on the substrate until the integral term would break the stiction, then flew until the distance to the closest waypoint shrunk so small that the next waypoint would be set as a target. At least, this was the expected behaviour. However, when breaking stiction, the magnetic force eventually was so large that the MagPier

This work was supported by $\mu$ RALP (FP7 ICT project no. 288663), Labex ACTION (ANR-11-LABX-01-01), MultiFlag (ANR-16-CE33-0019-02) and $\mu$ RoCS (ANR-17-CE19-xxxx-Xx). flew over the waypoint over to a further one. The controller being simple, not to say stupid, was therefore unable to stabilize the MagPier on the desired waypoint before switching to the next one. So, an ever more stupid motion controller was designed: "fly towards the next waypoint, update your position, determine which is the closest waypoint ahead on the sequence, and start again". And this stupid algorithm worked so well that the MagPier won the challenge twice in a row.

What did we do ? Actually, we rediscovered path following, a motion control strategy that is time independent and is essentially used for automated guided vehicles. It somehow differs with the motion strategies used in industrial robotics, where controller are defined with a dependence on time, as it will be explained in Section II. Turning later to biomedical applications, we found that path following could be profitably be used. Indeed, it allows to decouple laser tissue/interaction from anatomical constraints in laser surgery (Section III), it allows to derive a proper controller for microswimmers subject to non-holonomic constraints (Section IV) and it can even be coupled with remote center of motion (RCM) constraints in keyhole surgery (Section V).

The contribution of this paper is to trace our experience in path following at small scale through these examples, which explains the journalistic style used throughout the paper and the egocentrism of the references.

\section{Motion CONTROL STRATEGIES}

\section{A. Waypoint sequence}

The first motion control strategy consists in defining a sequence of intermediate positions (waypoints) to be reached by the robot as successive step responses: $\mathcal{S}=\left\{\mathbf{P}_{k}^{*} \in \Re^{3} \mid \forall k \in\{1, . . N\}\right\}$. More complex motion is achieved with a sequence over $S E(3)$ instead of $\Re^{3}$ to impose constraints on the orientation. It is thus extremely simple for the user. It is also very easily programmable since proportional control

$$
\mathbf{u}(t)=K_{p} *\left(\mathbf{P}(t)-\mathbf{P}_{k}^{*}\right)
$$

is often enough in the absence of strong disturbances if the system is linear or close to be linear. When disturbances occur, this controller is most often replaced by a PID controller, another linear controller. However, since the microrobot dynamics are often non-linear, tuning of the linear gains for 
any step might push those gains downwards. More important, this strategy provides a velocity profile, with exponentially decaying velocities from one waypoint to the other one. This profile is not really smooth, since the robots would alternately start and stop. This might even be troublesome for mobile microrobots in presence of friction.

To overcome these drawbacks, two classes of techniques can be found: interpolation between waypoints under velocity constraints (that gives rise to trajectory tracking) and appropriate accountance of differential geometry (that gives rise to path following).

\section{B. Trajectory tracking}

Trajectory tracking is the major motion control strategy in industrial robotics. A trajectory is defined by a set of $N$ (time, position) couples: $\left\{\left(t_{k}, \mathbf{P}^{*}\left(t_{k}\right)\right) \in \Re \times \Re^{3} \mid \forall k \in\{1, . . N\}\right\}$. Again, more complex trajectories can be defined over $S E(3)$ instead of $\Re^{3}$ to include orientation constraints. In general, a few number of such couples are defined, and then interpolation of various orders fills in the gap to densify the set: $\mathcal{T}=\left\{\mathbf{P}^{*}(t) \in \Re^{3} \mid \forall t \in \Re\right\}$ while preserving some smoothness property (usually $\mathcal{C}^{1}$ or $\mathcal{C}^{2}$ ) of the function $\mathbf{P}^{*}(t)$ with respect to time.

The advantages of this motion control strategy are many. Among others, it may provide optimality in the overall traversal time, it may take into account the robot dynamics and it is rather simple to implement with a proportional+feedforward controller:

$$
\mathbf{u}(t)=K_{p} *\left(\mathbf{P}(t)-\mathbf{P}^{*}(t)\right)+\frac{d \mathbf{P}^{*}(t)}{d t}
$$

Again, this controller can be extended to a PID+feedforward to account for small non-linearities and disturbances.

The drawbacks of this strategy are that it requires an expert roboticist for choosing the time constraints, whereas the nonroboticist user generally does not have the slightest hint on the latter. Moreover, specifically to low Reynolds swimming, where the swimming velocity varies with the fluid viscosity, such a control strategy is not robust to time delays and may thus create geometric deviations.

\section{Path following}

A path is defined as a smooth curve, at least $\mathcal{C}^{1}$, with respect to the distance $s \in \Re$ traveled along the curve: $\Gamma=\left\{\mathbf{P}(s) \in \Re^{3} \mid \forall s \in \Re\right\}$. It is thus independent from time. And so should the controller for its following be. Therefore, this motion control strategy decouples geometry from velocity, as a car can drive the same path on a motorway at various velocities.

The main drawback of path following is that it seems rather complex to implement. However, the advantages of it are many. From a technical viewpoint and by definition, path following is robust to time delays along the path and fully respects the desired geometric path. More important, it remains intuitive for the user to define the path (provided that the human-robot interface is adequately designed). From an application viewpoint, decoupling the geometric path from the velocity is particularly useful to biomedical applications of microrobotics. Indeed, for biological applications, respecting the geometric path is crucial to prevent contamination. For clinical applications, path-velocity decoupling garantees the respect of the surgical planning and allows the surgeon to share the control with the robot, the robot bringing accuracy and the surgeon keeping his hands on the tool progression.

\section{ENDOSCOPIC LASER STEERING}

The first example in this paper arised in the FP7 $\mu$ RALP project, dedicated to microrobot-assisted laser phonosurgery. In this context, the clinical specifications are that the surgeon would use the endoscopic image to delineate the tumor to be removed from the vocal folds with a laser and that the laser would move at a constant speed. Indeed, moving too slow, the laser would burn the tissues instead of incising them; moving too fast, it would simply have no effect.

Therefore, we decided to take advantage of the path-velocity decoupling within path following. Since the laser was observable in the endoscopic image, in which the desired path is defined by the surgeon, the problem was to drive a 2D point along a $2 \mathrm{D}$ path. A similar problem was already solved in the literature for automated guided vehicles, where an additional non-holonomic constraint is at stake: the vehicle can not move sideways. In order to have smooth motion of the laser (and to avoid zig-zaging scars), we deliberately applied the same nonholonomic constraint to the laser, although it was steered by a mirror and was physically not subject to such a constraint [3].

Thereby, the state of the laser spot is defined by $\left(s, d, \theta_{e}\right)$, where $s$ is the curvilinear abscissa of the closest point $\mathbf{h}_{p}$ to the laser spot on the path, $d$ is the shortest distance from the laser spot to the path and $\theta_{e}$ is the angle between the laser spot velocity $\mathbf{u}_{1}$ and the vector $\mathbf{x}_{s}$, tangent to the path in $\mathbf{h}_{p}$ (Figure 1).
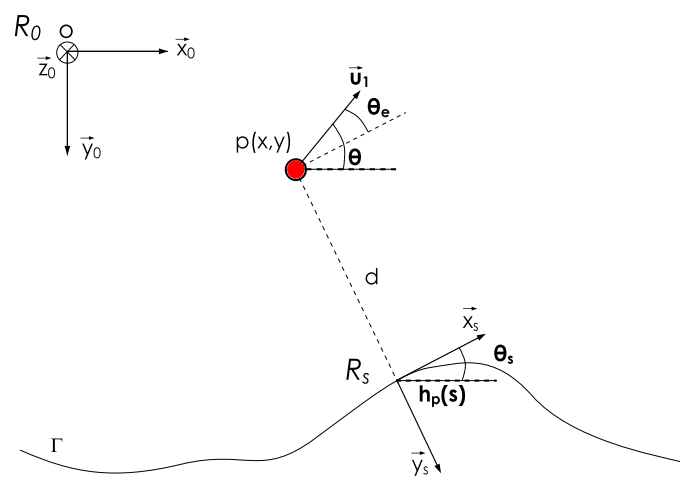

Fig. 1. Geometric description of the laser spot steering [3]

The virtual kinematics of the laser spot were then given by the unicycle kinematics:

$$
\begin{aligned}
\dot{s} & =\frac{u_{1}}{1-d C(s)} \cos \theta_{e} \\
\dot{d} & =u_{1} \sin \theta_{e} \\
\dot{\theta}_{e} & =u_{2}-\dot{s} C(s)
\end{aligned}
$$




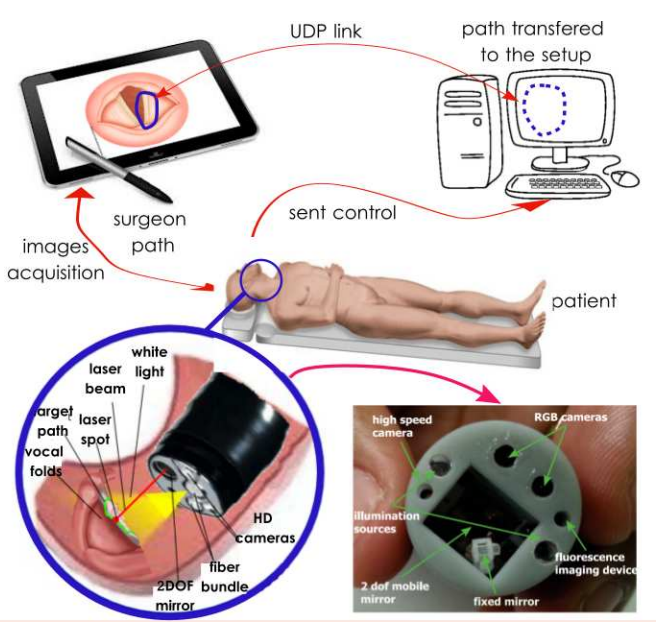

Fig. 2. Endoscopic Laser Surgery set-up [3]

where $u_{1}=\left\|\mathbf{u}_{1}\right\|$ is the norm of the laser spot velocity, $u_{2}=$ $\dot{\theta}_{e}$ is the norm of the angular velocity and $C(s)$ is the curvature of the path in $\mathbf{h}_{p}$. Note that $u_{1}$ and $u_{2}$ are the two control inputs, $u_{1}$ being dedicated to the laser spot velocity and $u_{2}$ being in charge to servo the laser spot on the path at the velocity given by $u_{1}$.

In that scheme, the non-roboticist user only needs to define the path $\Gamma$ and the laser velocity $u_{1}$. For instance, the former can be drawn with a stylet on a touchpad (Figure 2), while the latter can be intuitively controlled by foot pedals as in a car. It is the role of the developer to compute $u_{2}$ in function of the detected position of the laser spot in the image and those user inputs. The solution to this problem is elegantly given in [4] by an appropriate change of coordinates that casts the kinematics in a 3-state/2-inputs cascade system:

$$
\begin{aligned}
& \dot{z}_{1}=\dot{s} \\
& \dot{z}_{2}=\dot{s} z_{3} \\
& \dot{z}_{3}=v_{2}
\end{aligned}
$$

where $\dot{s}$ depends on the state and $u_{1}$ while $u_{2}$ can be deduced from the definition of $v_{2}$ in the change of coordinate:

$v_{2}=\left(-\dot{d} C(s)-d \frac{\partial C(s)}{\partial s} \dot{s}\right) \tan \theta_{e}+(1-d C(s))\left(1+\tan ^{2} \theta_{e}\right) u_{2}$

and from the stable control established for such a cascade system:

$$
v_{2}=-v_{1} \gamma_{2} z_{2}-\left|v_{1}\right| \gamma_{3} z_{3}
$$

where $\gamma_{2}$ and $\gamma_{3}$ are positive gains.

This control was associated to a parallel pan-tilt microrobot to move the laser and tested both on a testbed and in a human cadaver trial [5] with an accuracy of $30 \mu \mathrm{m}$ on the testbed and of $84 \mu \mathrm{m}$ in the preclinical test (Figure 3 ).

\section{MAGNeTiC MANiPUlation OF A MiCROSWIMMER}

Microswimmers, be they helical [6] or flagellar [7], [8], convert a time-varying uniform field field into motion thanks to their tail. This creates a non-holonomic constraint since the

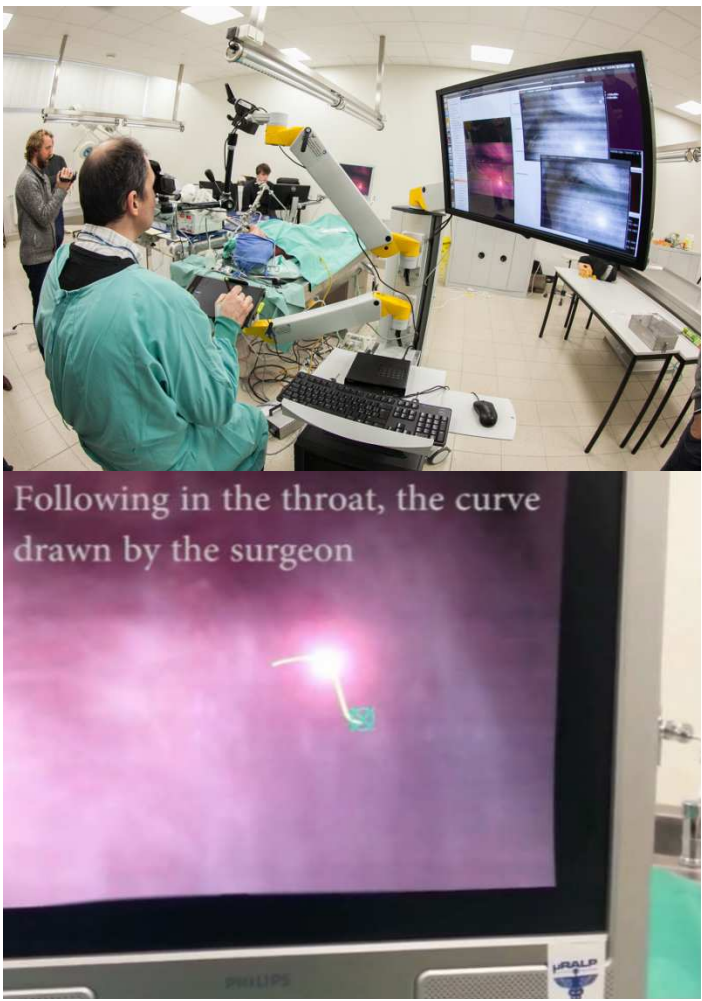

Fig. 3. Pre-clinical trial of endoscopic laser steering [5]

microswimmer can not move sideways. Moreover, the velocity amplitude of a helical swimmer can not be reliably achieved in many cases. The first reason for that is the well-known cutoff frequency of the velocity amplitude [9], which imposes a strong saturation of the available ampli- tude. As we have shown in [10], this cut-off frequency depends on many uncontrolled factors (microfabrication quality and environmental viscosity, for the main ones). The second reason is related to the potential stiction of the helix onto the substrate, which creates a distortion in velocity amplitude. The third reason is related to the viscosity at low scales (through the Reynolds number). Indeed, at low scales, we are not sure that the fluid is really homogeneous (inherently or due to the presence of micro or nano-dirt in a non totally clean room). This is especially true when dealing with biological fluids (blood, cell culture) where the medium is definitely heterogeneous or, at least, where the cells represent mobile obstacles that need to be pushed aside to allow for the helical swimmer to follow the expected trajectory.

For the non-roboticist user, the task for a microswimmer is usually to cargo a cell from one point to another one. However, with appropriate human-machine interface and with advanced control, the user will become able to define more complex tasks, such as navigating in a Petri dish through a complex map defined by several cell clusters separated by empty spaces. The non-holonomic constraint makes it natural to apply the controller defined in the previous section. This allowed us to accurately guide a helical swimmer in closed loop in a 


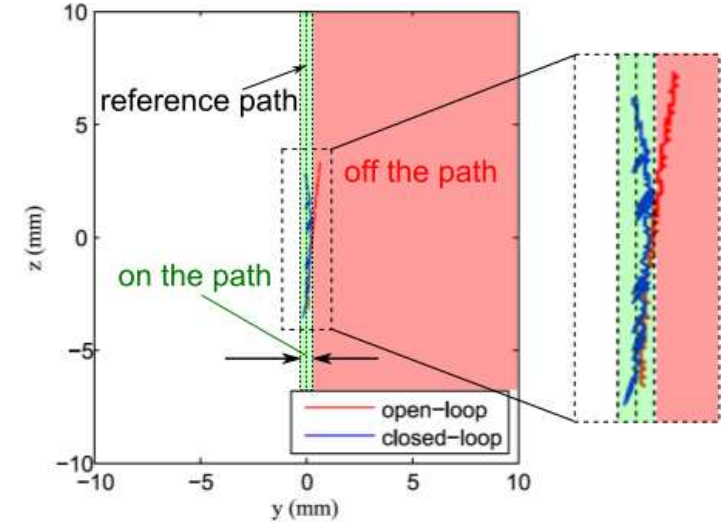

Fig. 4. Open-loop vs. closed loop control in planar path following [11]

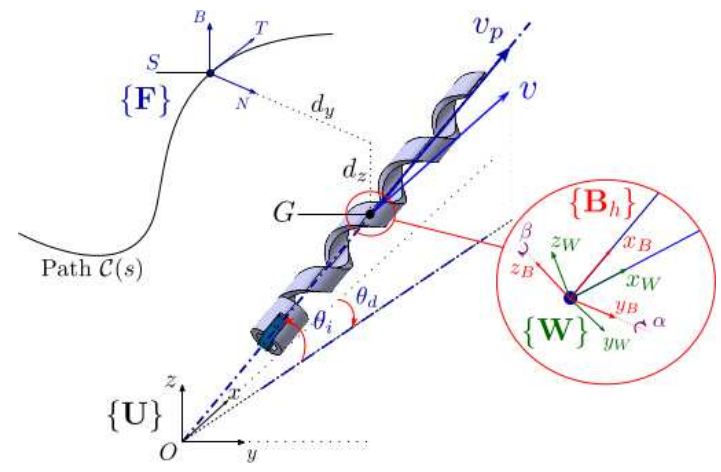

Fig. 5. Helical swimmer modelling [12]

horizontal plane (Figure 4), while the height of the swimmer was precompensated in open loop [11].

The planar controller was then extended to the 3D case, by extending the unicycle kinematics over a 5 degrees-offreedom state defined as $\left(s, d_{y}, d_{z}, \theta_{d e}, \theta_{i e}\right)$ where $s$ is the curvilinear abscissa of $\mathbf{h}_{p}$ (the orthogonal projection of the swimmer position onto the path), $d_{y}$ and $d_{z}$ are the projection of the distance from the swimmer to the path on two axes perpendicular to the path tangent vector, and finally $\theta_{d e}$ and $\theta_{i e}$ are the two angles defining the angular error between the swimmer velocity and the path tangent vector (Figure 5). Then, an appropriate change of coordinates allowed us to transform the swimmer model into a 5-state/3-inputs cascade system, from which we were able to derive a controller and prove the stability of the latter [12]. With that, we were able to follow a helix (Figure 6) with an accuracy better than $2 \%$ of the swimmer length [13]. Changing only the magnetic actuation mode but not the 3D path following controller [14], we were also able to navigate a swimmer with a flexible flagella along an arbitrary path (Figure 7).

\section{NAVIGATION OF A SURGICAL TOOL UNDER ANATOMICAL CONSTRAINTS}

The last example comes from another surgical context, this time in the case of middle-ear surgery. Again, the clinical specifications request accurate 3D path following for ablating
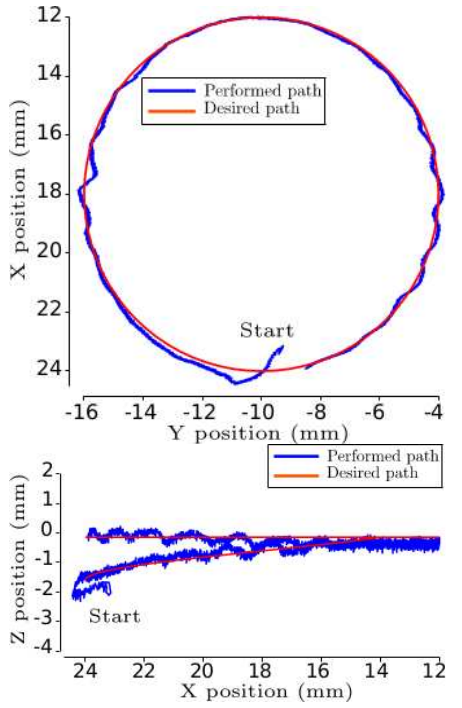

Fig. 6. Following a helix with a helical swimmer [12]

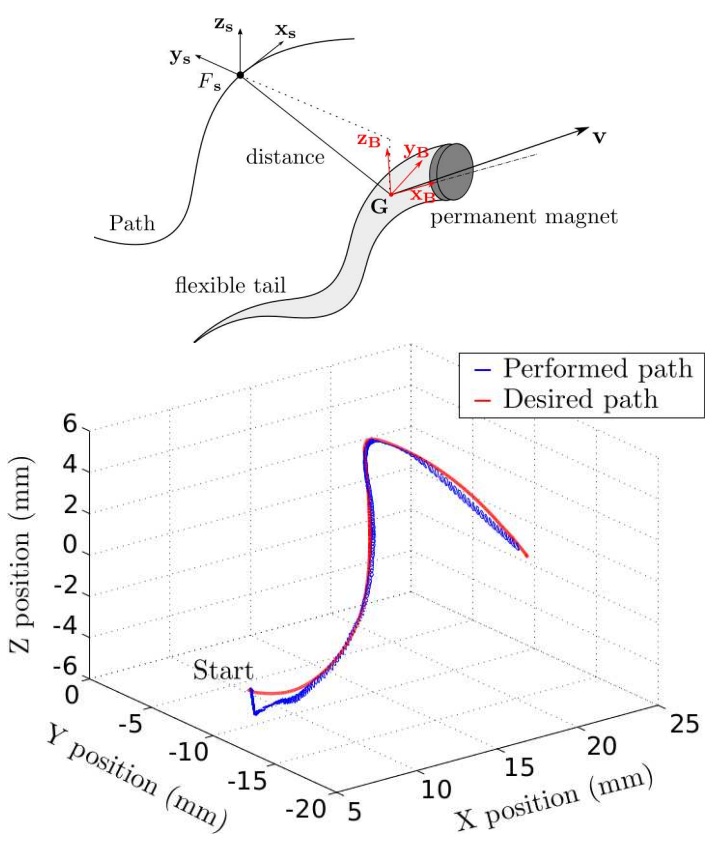

Fig. 7. Following an arbitrary path with a flexible flagella [14]

pathologic tissues around the ossicles. In addition, as in many other surgical case, the access to the middle ear is restricted to a single point while realizing the path following (Figure 8).

The definition of the 3D path can be done from any 3D surgical planner. However, the specifications do not mention any constraint on the velocity. In fact, the exact time to finish the path is not a factor of significant nature for the surgeon. What really matters is that the instrument follows exactly the intended path. The surgeon may also need to change the tool velocity independently from the path shape, size or curvature. The instrument velocity is indeed dependent on the tissue-tool interaction which could be variable due to the potential inhomogeneity of the tissue. In addition, the tool 


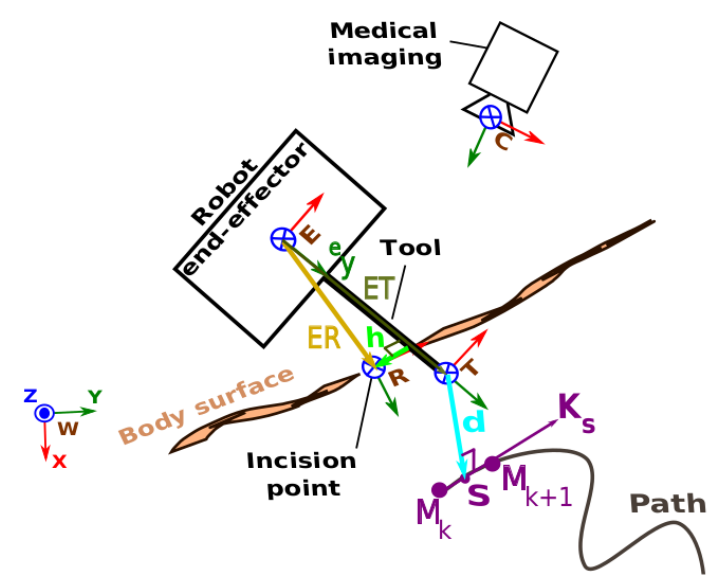

Fig. 8. Path following under single point access constraint [15]

velocity profile may change when the surgeon scans a ROI, or moves from one ROI to another for inspection. For the above reasons, the path following controller is a good option for our application; since it separates the geometric curve from the velocity profile.

Note also that this is coherent with surgical planners. Indeed, as far as we know, surgical planners restrict their work to path definition and do not time stamp the path into a trajectory.

On the contrary to the first two examples, non-holonomic constraints were neither physically imposed nor virtually chosen. Only the position of the tool tip was considered in the control. Thereby, the state of the system is given by $(s, \mathbf{d})$ where $\mathbf{d}=\mathbf{p}-\mathbf{h}_{p}$ is the vector, not scalar, distance from the path to the tool tip. After geometric analysis in $3 \mathrm{D}$, the time derivative of the lateral error becomes;

$$
\dot{\mathbf{d}}=\left(\mathbf{I}_{3 \times 3}-\frac{\mathbf{x}_{s} \mathbf{x}_{s}^{T}}{1-\mathbf{d}^{T}\left(\mathbf{C}(s) \times \mathbf{x}_{s}\right)}\right) \mathbf{u}_{1}
$$

where $\mathbf{C}(s)$ is the path curvature vector. As a consequence, a Lyapunov-stable controller was proposed [15] as:

$$
\mathbf{u}_{1}=\alpha \mathbf{x}_{s}+\beta \mathbf{d}
$$

where $\alpha$ depicts the tangential velocity and $\beta$ is a control gain for correcting the lateral error. Since $\left\|\mathbf{u}_{1}\right\|$ is controlled by the surgeon, $\alpha$ and $\beta$ are not independent from each other. It turned out that a good strategy is to make $\alpha$ dependent on $\beta$ since the surgical constraint is to respect the path rather than the progression along it. As a consequence, $\alpha$ was chosen as:

$$
\alpha= \begin{cases}\sqrt{\left\|\mathbf{u}_{1}\right\|^{2}-\beta^{2}\|\mathbf{d}\|^{2}} & \text { if } \beta^{2}\|\mathbf{d}\|^{2}<\left\|\mathbf{u}_{1}\right\|^{2} \\ 0 & \text { else }\end{cases}
$$

With that, priority is given to the reduction of the lateral error and then, if possible, to progress along the path. Experiments on a real robot (Figure 9) achieved a path following error of some $300 \mu \mathrm{m}$ while maintaining the distance of the tool body to the incision point within $300 \mu \mathrm{m}$, in a suboptimal hardware set-up.

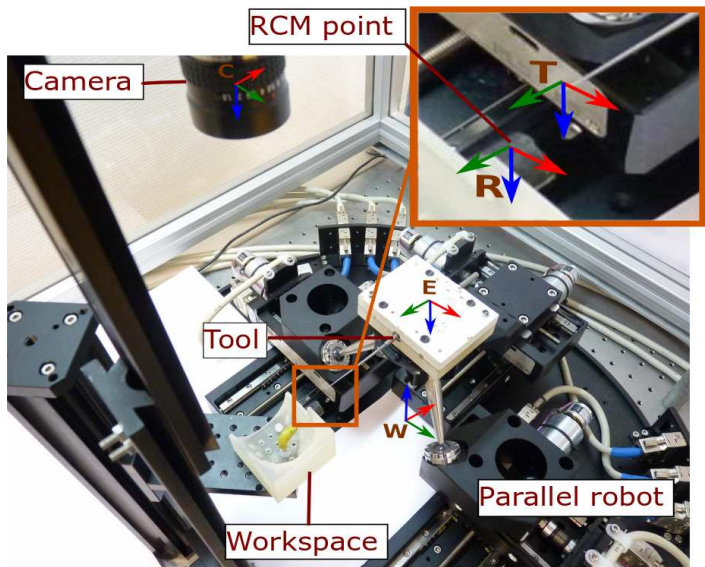

Fig. 9. Set-up for path following under single port access constraint

\section{CONCLUSION}

Through this paper, several examples of path following in the domain of microrobotics were reported. We tried to explain for all cases, the user requirements and why decoupling path following in a closed-loop control from the manual or openloop control of the velocity along the path was relevant. Short technical descriptions and some results were given, as an appetizer for reading the full papers and converting to path following in microrobotics.

\section{REFERENCES}

[1] N. Chaillet and S. Régnier, Microrobotics for Micromanipulation. Wiley, 2010.

[2] I. A. Ivan, G. Hwang, J. Agnus, N. Chaillet, and S. Régnier, "Nist and ieee challenge for magpier: The fastest mobile microrobots in the world," IEEE Robotics Automation Magazine, vol. 20, no. 2, pp. 63-70, June 2013.

[3] J. Séon, B. Tamadazte, and N. Andreff, "Decoupling path following and velocity profile in vision-guided laser steering," IEEE Transactions on Robotics, vol. 31, no. 2, pp. 280-289, Apr. 2015.

[4] P. Morin and C. Samson, "Motion control of wheeled mobile robots," in Springer Handbook of Robotics, B. Siciliano and O. Khatib, Eds., 2008, pp. $799-826$.

[5] R. Renevier, B. Tamadazte, K. Rabenorosoa, N. Andreff, and L. Tavernier, "Endoscopic laser surgery: Design, modeling and control," IEEE Transactions on Mechatronics, vol. 22, no. 1 , pp. 99-106, Feb. 2017. [Online]. Available: https://publiweb.femtost.fr/tntnet/entries/13611/documents/author/data

[6] S. Tottori, L. Zhang, F. Qiu, K. Krawczyk, A. Franco-Obregn, and B. Nelson, "Magnetic helical micromachines : Fabrication, controlled swimming, and cargo transport," Advanced Materials, vol. 24, no. 6, pp. 811-816, Feb. 2012.

[7] I. Khalil, K. Youakim, A. Sanchez, and S. Misra, "Magnetic-based motion control of sperm-shaped microrobots using weak oscillating magnetic fields," in IEEE/RSJ International Conference on Intelligent Robots and Systems, Chicago, 2014, pp. 4686-4691.

[8] Z. Ye, S. Régnier, and M. Sitti, "Rotating magnetic miniature swimming robots with multiple flexible flagella," IEEE Trans. Robotics, 2013.

[9] T. Honda, K. Arai, and K. Ishiyama, "Micro swimming mechanisms propelled by external magnetic fields," IEEE Transactions on Magnetics, vol. 32, no. 5, pp. 5085-5087, Sep. 1996.

[10] T. Xu, G. Hwang, N. Andreff, and S. Régnier, "Modeling and swimming property characterizations of scaled-up helical microswimmers," IEEE/ASME Transactions on Mechatronics, vol. 19, no. 3, pp. 10691079, 2013.

[11] _ - "Planar path following of $3 \mathrm{~d}$ steering scaled-up helical microswimmers," IEEE Transactions on Robotics, vol. 31, no. 1, pp. 117127, 2015. 
[12] A. Oulmas, N. Andreff, and S. Régnier, "Chained formulation of 3D path following for nonholonomic autonomous robots in a Serret-Frenet frame," in American Control Conference, Oct. 2016, pp. 7275-7280.

[13] _ , "Closed-loop 3D path following of scaled-up helical microswimmers," in IEEE/RSJ International Conference on Intelligent Robots and Systems, Oct. 2016, pp. 1725-1730.

[14] _ "3D closed-loop motion control of swimmer with flexible flagella at low Reynolds numbers," in IEEE/RSJ International Conference on Intelligent Robots and Systems, 2017.

[15] B. Dahroug, B. Tamadazte, and N. Andreff, "Visual servoing controller for time-invariant $3 \mathrm{D}$ path following with remote centre of motion constraint," in Robotics and Automation (ICRA), 2017 IEEE International Conference on. IEEE, 2017, pp. 3612-3618. 